\title{
Copyright Protection of Images by reversible, invisible, and robust Digital Watermarking using 3rd and 4th Level Discrete Wavelet Transform
}

\author{
Mayank Mishra $^{\# 1}$, Dr. Nirmal Kumar Rout ${ }^{* 2}$ \\ \# M. Tech Scholar, School of Electronics Engineering, KIIT University, Bhubaneswar, Odisha, India. \\ ${ }^{1}$ mmishra1208@gmail.com \\ * Professor, School of Electronics Engineering, KIIT University, Bhubaneswar, Odisha, India. \\ ${ }^{2}$ routnirmal@rediffmail.com
}

\begin{abstract}
With the rapid development in the digital world, the authentication and protection of the data i.e. audio, image, video, is one of the major problem in the present era. Digital watermarking is the technique to provide copyright protection of the data. In past and in recent years many algorithms or schemes have been introduced for copyright protection of images. Among which few are unable to recover the original image after embedding the watermark i.e. the image get distorted during extraction of watermark. And few schemes are reversible in nature. In this paper, we proposed a watermarking scheme in discrete wavelet transform (DWT) domain, which is not only reversible in nature but also robust against various attacks in providing copyright protection of images. In the proposed scheme, watermark is embedded at third and fourth level DWT and extracted watermark is compared with original watermark. Watermarked image is attacked by various types of attacks to check the invisibility, robustness \& effectiveness of the scheme. Watermark is extracted from the attacked watermarked image and then compared with original watermark based on normalized correlation to validate the proposed scheme of providing reversibility in digital watermarking. It is useful in various real time applications such as: copyright protection, broadcast monitoring, content archiving, digital fingerprinting, etc.
\end{abstract}

Keywords— Digital watermarking, 3-Level DWT, 4-Level DWT, Discrete wavelet transform, copyright protection.

\section{INTRODUCTION}

In comparison to traditional analog media, digital media is becoming more popular with invention of high speed processor. Digital media can be stored, duplicated and distributed without loss of fidelity unlike analog media [1]. Digital content that have been used in a network environment are easy to be duplicated, so enforcement of digital copyright protection is an important issue [2]. Digital watermarking solves the problem of copyright protection by watermark embedding in digital data, such as image [3]. The watermark embedded in digital works must have the following important features to be called digital watermarking:

1) Invisibility: The process of digital watermark embedding in the image does not distort the quality of image. The embedded watermark must be invisible after the embedding process in the image. Generally, in practise our subjective feeling is used to observe the invisibility of the embedded watermark. We can assume the watermark transparency and invisibility by our human senses, whether it contains a watermark or not.

2) Stability (robustness): Digital signals sometime go through intentionally or unintentionally transformation like filtering, noises and many attacks during dissemination processes. The embedded information i.e. watermark must have some stability against such operations or transformations.

3) Security: Anti-interference ability should be possessed by digital watermark against various attacks, due to which unauthorised person may find large difficulty in copying or modifying it. In other words, any third person who is not authorised to access the data i.e. image, cannot read and detect the embedded information.

4) Effectiveness: Effectiveness of the scheme or algorithm for watermark extraction will be validated only when, it will be simple and fast [4].

As per the domain in which the embedding of watermark will be done, the watermarking techniques are classified into two categories such as: spatial domain techniques and transform domain techniques [5-6, 9-15, 19-20]. In comparison to transform domain techniques, the spatial domain techniques are simple but they are very less robust against many attacks [5-6]. Some transform domain techniques used for watermarking are discrete cosine transform (DCT), discrete wavelet transform (DWT), discrete Fourier transform (DFT), etc. In 
comparison to spatial domain algorithm, the watermark information embedded in the transform domain more evenly distributed, by spreading to all the pixels in the original image, which will be favourable in ensuring the invisibility of the watermark. In transform domain algorithm the more information can be embedded without degrading the quality of the image. Watermark algorithm based on transform domain has many advantages in comparison to spatial domain because of its excellent performance [4]. Among the various transform domains techniques, wavelet based techniques are more popular because of its excellent frequency localisation properties [5]. Digital watermarking technology involves various image processing concepts and mathematical tools. It becomes very complex situation while using the ordinary programming tools for the implementation of the schemes. So MATLAB is one of the best tools for the digital watermarking scheme [8]. Many researchers in different fields are now using digital watermarking technique in various applications [21]. In past and in recent years many algorithms or schemes have been introduced for copyright protection of images [1-6, 22-27]. Among which few are unable to recover the original image after embedding the watermark i.e the image get distorted during extraction of watermark [6]. All these algorithms have various advantages with some limitations. In the proposed scheme, watermark is embedded at third and fourth level DWT to increase the level of security, so that it is difficult for unauthorized person to detect the embedded watermark along with its level and subband of the DWT. In this paper performance of 3rd and 4th level of the DWT reversible digital watermarking is evaluated. The very first and important rule of digital watermarking is its invisibility feature. For its justification, histogram of both host and watermarked images are compared on the basis of distribution of various pixels of various intensities. Also, it is observed that watermark is invisible after embedding in host image by human perception of visualization. The invisibility criteria are not the only features required in digital watermarking. Second feature is the reversibility. For the validity of the reversibility feature, the extracted watermark image is compared with the original watermark image on the basis of normalized correlation (NC) value. If the $\mathrm{NC}$ value between the watermarked and the host image is equal to 1 , then the watermark embedded in the particular level of the DWT is reversible and not distorted during its extracting process from the watermarked image. Robustness or stability against various attacks is one of the most important required features in digital watermarking technique. The watermarked image is attacked by various types of standard attacks. Then watermark is extracted from attacked watermarked image and compared with original watermark based on $\mathrm{NC}$ value to validate the proposed scheme of providing robustness in digital watermarking. If $\mathrm{NC}$ value is greater than 0.5 it shows the similarity between two images. Maximum value of NC can be 1 . It is very much essential in various real time applications.

\section{DISCRETE WAVELET TRANSFORM}

The Discrete wavelet transform depends on wavelets. It is helpful in observing the image in various frequency components at distinct frequency levels. Discrete wavelet transform (DWT) is a multi-resolution representation of an image [7].

The DWT can be implemented as a multistage transformation. Wavelet transform divides the image into horizontal, vertical, diagonal, and low frequency subbands. Low frequency part which contains the maximum information of the image, known as the approximation subband and the other parts are called the detail subband. At first level in the DWT an image is divided into four sub-bands denoted as LL, LH, HL, and HH domain, where LH, HL, and HH symbolizes the high frequency wavelet coefficients and LL symbolize low frequency wavelet coefficients. The decomposition process continues in the LL subband as per the required application. To maintain better image quality, high frequency subbands are more suitable for watermark embedding, as human eyes are much more sensitive to the low-frequency part (the LL sub-band) $[16,19]$.

Process of wavelet decomposition can be expressed in the following form: [17]

$$
c M_{t}=c M_{t+1}+c H_{t+1}(x)+c H_{t+1}(y)+c H_{t+1}(z)
$$

where $c M$ shows the low frequency part i.e. approximation part of the image and $c H(x), c H(y)$ and $c H(z)$ show the higher frequency parts i.e. horizontal, vertical and diagonal part of the image in higher frequency region. The variable ' $t$ ' represents the level of decomposition. In general, we represent high frequency bands like HH, LH and HL subbands. For further steps, there will be decomposition of $c M_{t}$ as $c M_{t+1}$ and so on as per the applications. The large coefficients normally indicate edges in the image in these bands. Therefore, the DWT understands the human visual system [18].

\section{THE PROPOSED SCHEME}

The proposed scheme is elaborated in this section. In general, a scheme of watermarking includes two main stages such as watermark embedding stage and watermark extraction stage. The proposed scheme includes both the stages to provide invisibility, reversibility and robustness features. The proposed scheme has used grayscale 
images. One image is used as host image and other one as watermark image. It is found from the reported literatures that the. LL subband is not suitable for watermark embedding. As the LL subband is a low frequency subband and it contains maximum information of the image which is distorted by embedding the watermark in this subband. The HH subband contributes insignificantly towards signal energy and contains finer details. Hence, embedding of watermark in this region will not affect the perpetual fidelity of the image [11]. In the proposed scheme, watermark is embedded at third and fourth level DWT to increase the level of security, so that it is difficult for unauthorized person to detect the embedded watermark along with its level and subband of the DWT.

\section{A. DWT at 3rd and 4th level}

In proposed scheme the embedding of watermark is done in the HH subband in 3rd and 4th level of the DWT as shown in the Fig. 1 and Fig. 2 respectively. The proposed scheme is described in the section B. To check the invisibility feature of the scheme, histogram of both host and watermarked images are compared on the basis of distribution of various pixels with their different intensities and also by human perception of visualization. It is observed that watermark is invisible after embedding in the host image. For validation of reversibility feature, watermark is extracted and compared with original watermark. And to justify the robustness feature of the scheme, watermarked image gone through various attacks, like salt and pepper noise, speckle noise, histogram equalization, motion blur, gamma intensity transformation, scaling (multiply and divide), etc.

\section{B. Steps involved in the proposed scheme}

The proposed scheme can be described as follows:

Step1:The grayscale original image i.e. host image decomposes by the DWT till 3rd level for one case and 4th level for second case.

Step2: Other gray scale image taken as watermark, and is embedded in HH sub-band of 3rd/4th level of DWT by considering key $(\mathrm{K})$.

Step3:To check the stability of the proposed scheme watermarked image is gone through various attacks, which causes some distortion in the watermarked image.

Step4:Watermark is extracted from the attacked watermarked image.

Step5:The extracted watermark image is compared with the original watermark image on the basis of normalized correlation (NC). If NC value closer to 1, it shows the robustness of the scheme.

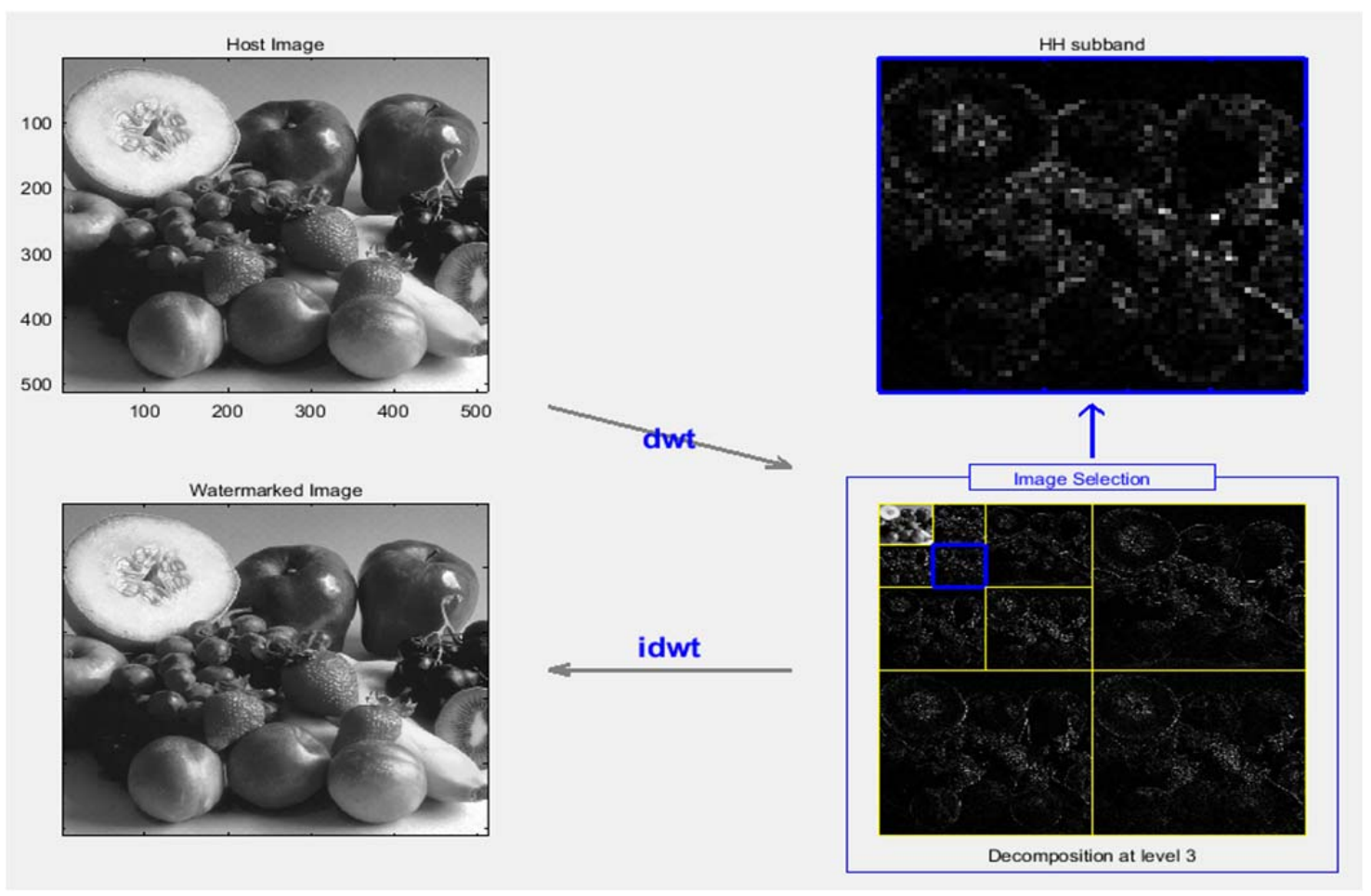

Fig.1. DWT of host image at 3rd level 


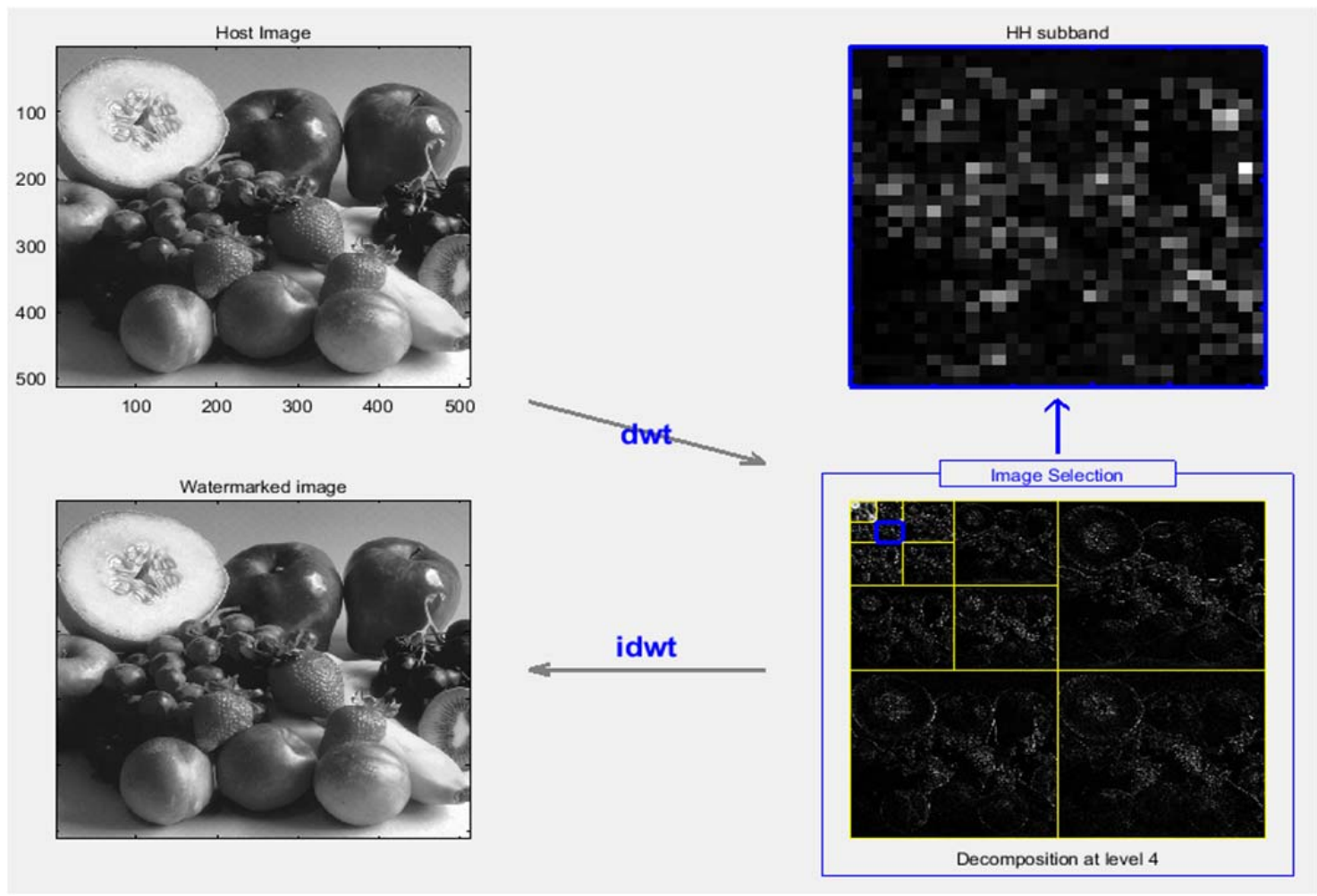

Fig. 2. DWT of host image at 4 th level

\section{RESULT AND DISCUSSION}

The proposed scheme is tested on grayscale host image of size 512 x 512 pixels and watermark image of size $64 \times 64$ pixels for 3rd level DWT and $32 \times 32$ pixels for 4th level DWT. After DWT at 3rd and 4th level, watermark is embedded in the $\mathrm{HH}$ subband of that particular level using the key. After the watermark embedding stage, watermark extraction stage is performed as it is the only symbol to authenticate the copyright protection of the image and to show the reversibility. The Haar wavelet is used for the DWT in the proposed schemes. All the stimulation is performed in the MATLAB-R2015a. After watermark embedding, the important analysis, i.e., invisibility and robustness analysis are performed to validate the proposed scheme in providing the copyright protection to the images.

\section{A. Invisibility and reversibility analysis}

Invisibility feature of proposed scheme is validated, as the watermarked and original host image seems to be similar as shown inFig3, Fig 5 and Fig 8. It shows that there is no visual difference between original watermark and extracted watermark, as both the images i.e. host and watermarked are looking similar as per the human visualisation. For further justification of invisibility feature, the histogram between host and watermarked image for both 3rd and 4th level of DWT is found to be similar as shown in Fig 10-12.

For reversibility analysis, extracted watermark shown in Fig 6 and Fig 9 is compared with original watermark shown in Fig 4 and Fig 7 respectively with the help of the NC values. The NC values can be computed between original watermark and extracted watermark as follows:

$$
\text { Normalised Correlation }(N C)=\frac{\sum_{x=0}^{D-1} \sum_{y=0}^{D=1} W(x, y) \cdot W_{e}(x, y)}{\sum_{y=0}^{D-1}[W(x, y)] \cdot[W(x, y)]}
$$

where $W(x, y)$ and $W_{e}(x, y)$ are the original and extracted watermark respectively, $\mathrm{D}$ is the dimension of original and extracted watermark image, $(\mathrm{x}, \mathrm{y})$ are the coordinate of pixels of the original and extracted watermark. The Table I gives the information of NC values between extracted watermark and original watermark which is equals to 1 for both 3rd and 4th level DWT. It shows the watermark embedded in the particular level of DWT, is reversible and not distorted during its extraction process from watermarked image. 


\section{B. Robustness analysis}

To validate this feature, watermarked image is attacked by various types of attacks such as contrast attack, salt and pepper noise attack, scaling (multiply and divide) attack, speckle noise attack, sharpening attack, motion blur attack, histogram equalization attack, Gaussian noise attack, gamma intensity transformation attack, wavelet transform attack (1 Level DWT). For the evaluation of robustness of the proposed scheme, all these attacks are applied on the watermarked image at 3rd/4th Level DWT, which produces distortion in the watermarked image. The attacked watermarked image and extracted watermark image at 3rd and 4th level DWT is shown in the Fig 13-34. Again the extracted watermark from the attacked watermarked image is compared with original watermark based on the $\mathrm{NC}$ values. To check the similarity between original watermark and extracted watermark, the $\mathrm{NC}$ value is calculated.

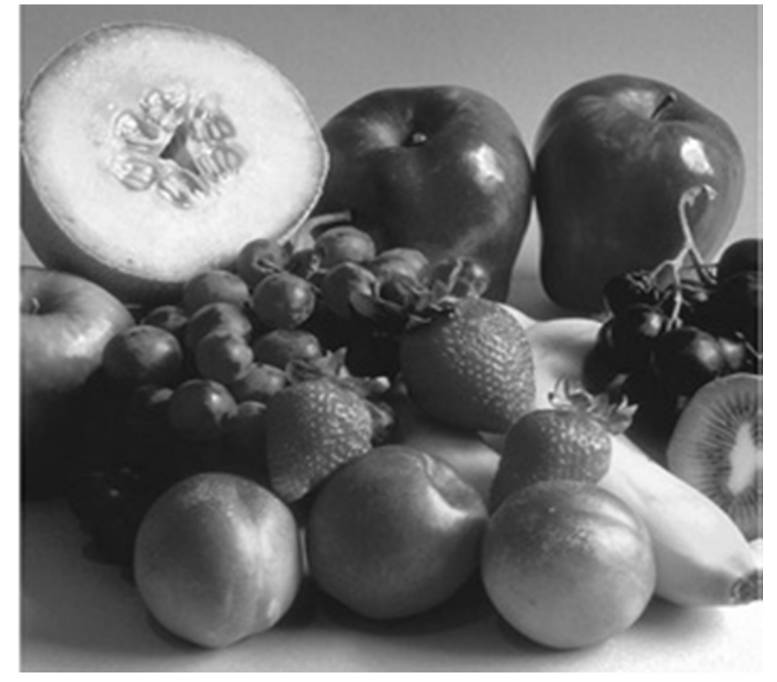

Fig. 3. Host Image

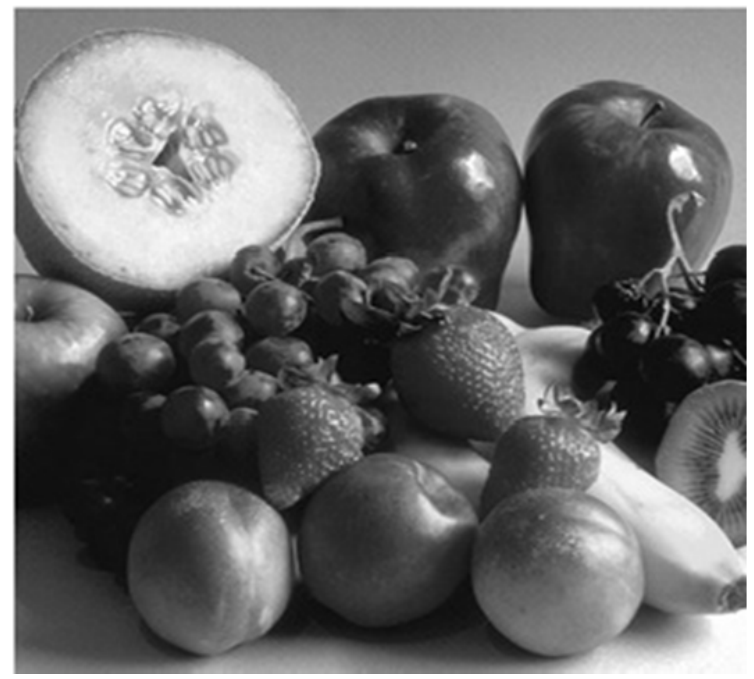

Fig. 5. Watermarked Image (3rd level DWT)
Fig . 4. Original watermark ( 3 rd level DWT)

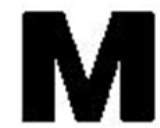

Fig . 6. Extracted watermark ( 3rd level DWT)

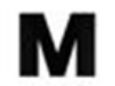

Fig. 7. Original watermark (4th level DWT)

The Table II compares the proposed scheme at 3rd and 4th level of DWT on the basis of NC values. Experimental results show the proposed scheme is robust against various attacks, as NC values are closer to 1 in every attack. Experimental results validate the proposed scheme in providing invisibility, reversibility and robustness in digital watermarking for copyright protection of images that is beneficial in various real time applications. The experimental results also concludes, the proposed scheme is more robust at 4th level of DWT in comparison to 3rd level DWT. 

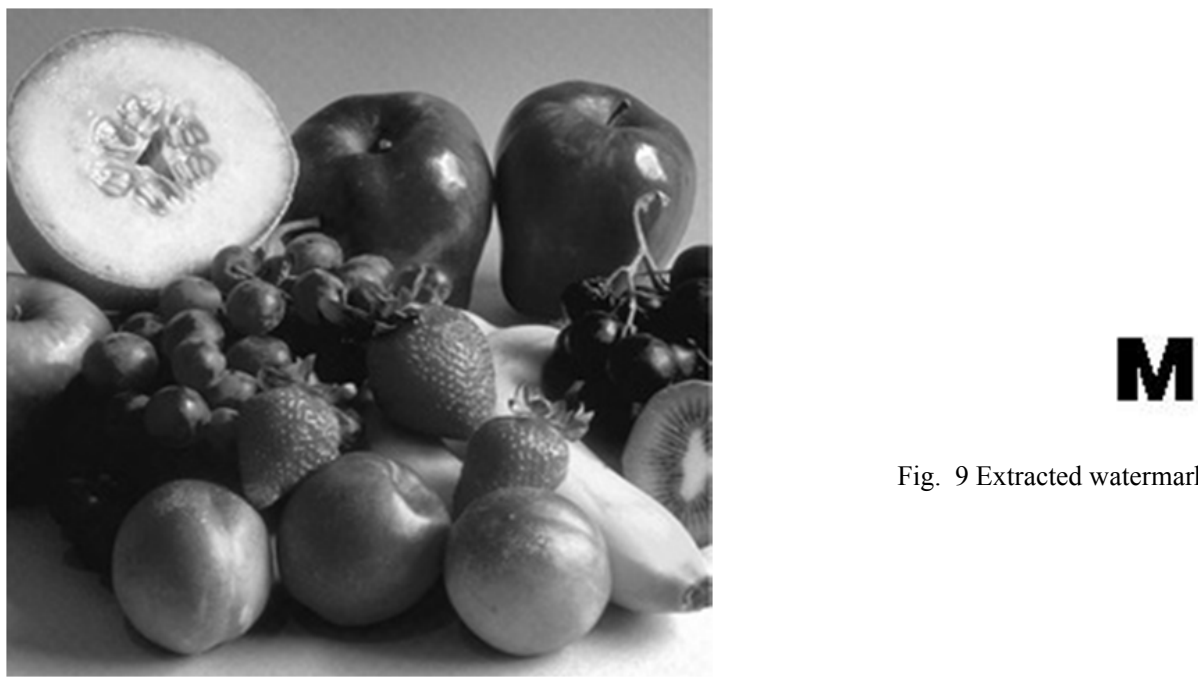

Fig. 9 Extracted watermark (4th level DWT)

Fig. 8. Watermarked Image (4th level DWT)
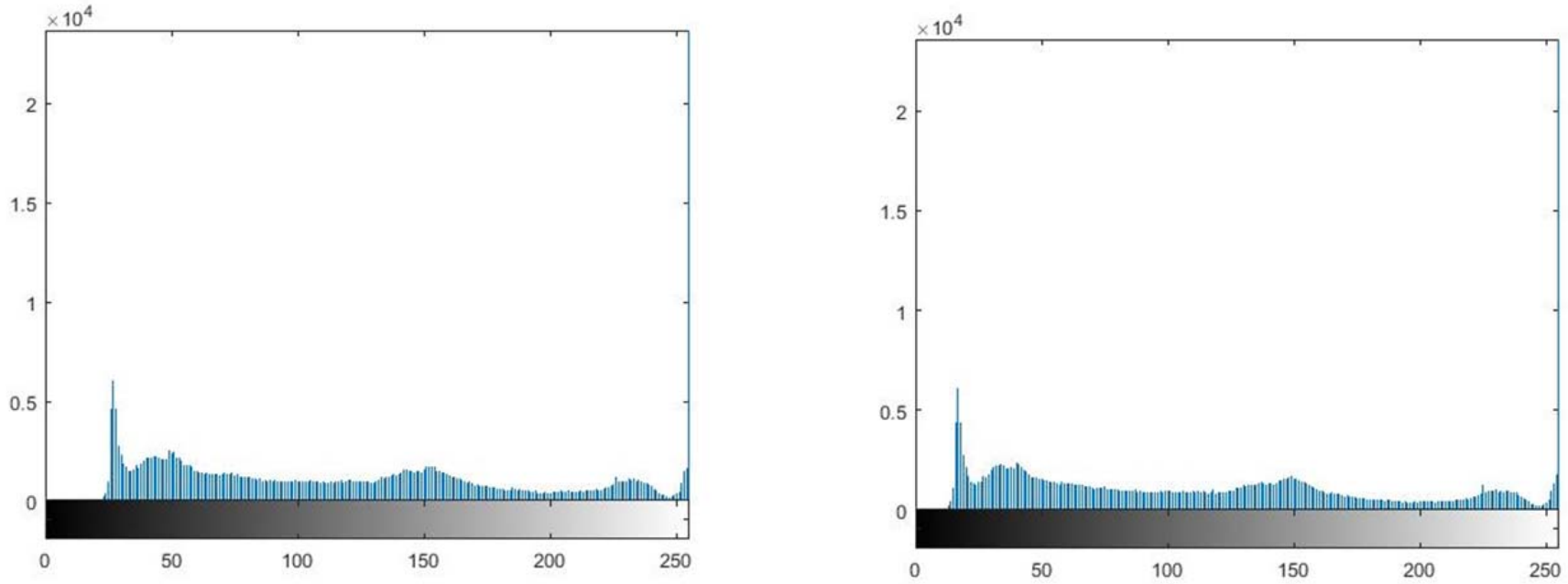

Fig. 10. Histogram of Host Image

Fig. 11. Histogram of Watermarked Image (3rd level DWT)

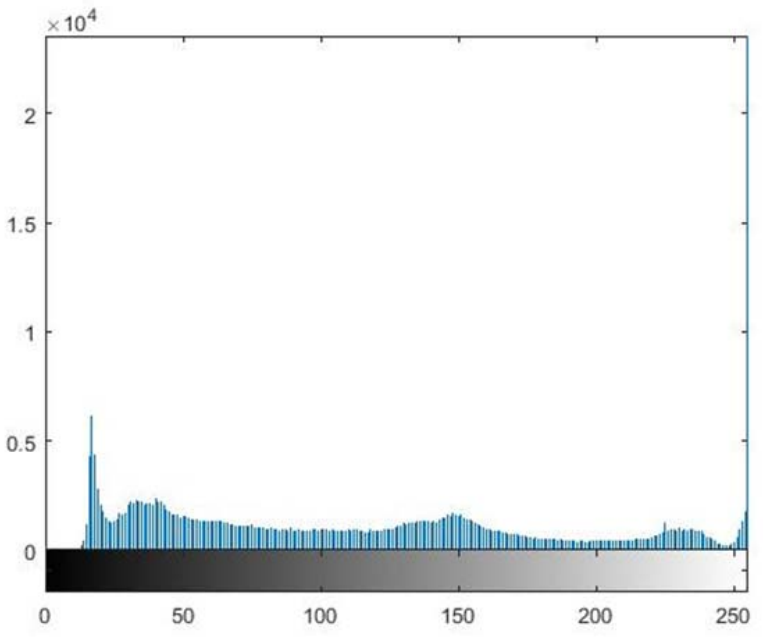

Fig. 12. Histogram of Watermarked Image (4th level DWT) 
TABLE I

NC VALUE AT 3RD AND 4TH LEVEL DWT BETWEEN

ORIGINAL WATERMARK AND EXTRACTED WATERMARK

\begin{tabular}{|c|c|}
\hline Level & Normalised Correlation \\
\hline 3rd & 1 \\
4 th & 1 \\
\hline
\end{tabular}

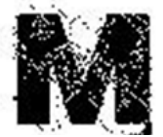

Fig. 13. Extracted watermark after contrast attack (3rd level DWT)

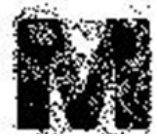

Fig. 15. Extracted watermark after scaling attack (multiplied with constant $=0.8,3$ rd level DWT)

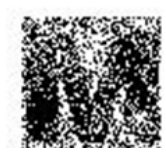

Fig. 17. Extracted watermark after speckle noise attack (variance $=0.01$, 3rd level DWT)

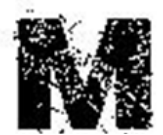

Fig. 19. Extracted watermark after motion blur attack (length $=2$ pixel and angle $=95^{\circ}, 3$ rd level DWT)

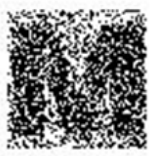

Fig. 21. Extracted watermark after Gaussian noise attack (variance $=0.001,3$ rd level DWT)
Fig. 14. Extracted watermark after salt and pepper attack $($ density $=0.015,3$ rd level DWT)

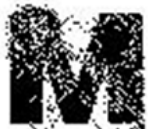

Fig. 16. Extracted watermark after scaling attack (divided by constant $=0.8,3$ rd level DWT)

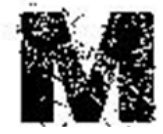

Fig. 18. Extracted watermark after sharpening attack (3rd level DWT)

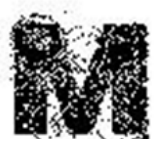

Fig. 20. Extracted watermark after histogram equalization attack (3rd level DWT)

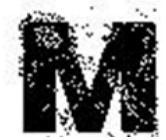

Fig. 22. Extracted watermark after gamma intensity transformation attack (gamma $=0.7,3$ rd level DWT) 


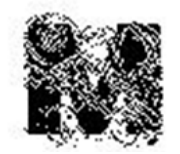

Fig. 23. Extracted watermark after DWT attack (level=1, 3rd level DWT)

Fig. 25. Extracted watermark after salt and pepper noise attack (density $=0.015,4$ th level DWT)

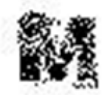

Fig. 27. Extracted watermark after scaling attack (divide by constant $=0.8,4$ th level DWT)

\section{M}

Fig. 29. Extracted watermark after sharpening attack (4th level DWT)

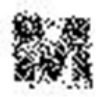

Fig. 31. Extracted watermark after histogram equalization attack (4th level DWT)

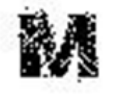

Fig. 33. Extracted watermark after gamma intensity transformation attack (gamma $=0.7,4$ th level DWT)

\section{菒}

Fig. 24. Extracted watermark after contrast attack (4th level DWT)

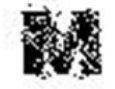

Fig. 26. Extracted watermark after scaling attack (multiplied with constant $=0.8$, 4 th level DWT)

Fig. 28. Extracted watermark after speckle noise attack (variance $=0.01,4$ th level DWT)

\section{M}

Fig. 30. Extracted watermark after motion blur attack (length $=2$ pixel and angle $=95^{\circ}, 4$ th level DWT)

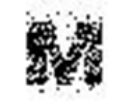

Fig. 32. Extracted watermark after Gaussian noise attack (variance $=0.001,4$ th level DWT)

Fig. 34. Extracted watermark after DWT attack (level=1, 4th level DWT) 
TABLE II

COMPARISON BETWEEN 3RD AND 4TH LEVEL DWT WATERMARKING ON THE BASIS OF NC VALUES BETWEEN ORIGINAL WATERMARK AND EXTRACTED WATERMARK

\begin{tabular}{|c|c|c|c|c|}
\hline \multicolumn{3}{|c|}{ Attacks } & NC value at 3rd level DWT & NC value at 4th level DWT \\
\hline \multicolumn{3}{|c|}{ Contrast } & 0.9161 & 0.9442 \\
\hline \multirow{3}{*}{$\begin{array}{c}\text { Salt and } \\
\text { pepper noise }\end{array}$} & \multicolumn{2}{|c|}{ Density $=0.01$} & 0.7831 & 0.8070 \\
\hline & \multicolumn{2}{|c|}{ Density $=0.015$} & 0.7365 & 0.7791 \\
\hline & \multicolumn{2}{|c|}{ Density $=0.02$} & 0.6765 & 0.7186 \\
\hline \multirow{3}{*}{$\begin{array}{l}\text { Scaling } \\
\text { (Multiply) }\end{array}$} & \multicolumn{2}{|c|}{ Constant $=0.09$} & 0.9342 & 0.9488 \\
\hline & \multicolumn{2}{|c|}{ Constant $=0.085$} & 0.8940 & 0.9140 \\
\hline & \multicolumn{2}{|c|}{ Constant $=0.08$} & 0.8309 & 0.8884 \\
\hline \multirow{3}{*}{$\begin{array}{l}\text { Scaling } \\
\text { (Divide) }\end{array}$} & \multicolumn{2}{|c|}{ Constant $=0.09$} & 0.9332 & 0.9465 \\
\hline & \multicolumn{2}{|c|}{ Constant $=0.085$} & 0.8897 & 0.9070 \\
\hline & \multicolumn{2}{|c|}{ Constant $=0.08$} & 0.8701 & 0.8674 \\
\hline \multirow{3}{*}{ Speckle Noise } & \multicolumn{2}{|c|}{ Variance $=0.01$} & 0.6324 & 0.8512 \\
\hline & \multicolumn{2}{|c|}{ Variance $=0.015$} & 0.6183 & 0.7884 \\
\hline & \multicolumn{2}{|c|}{ Variance $=0.02$} & 0.6040 & 0.7581 \\
\hline \multicolumn{3}{|l|}{ Sharpening } & 0.9540 & 0.9977 \\
\hline \multirow{8}{*}{ Motion Blur } & \multicolumn{4}{|c|}{ Length $=2$} \\
\hline & Angle & $90^{\circ}$ & 0.8370 & 0.9977 \\
\hline & Angle & $95^{0}$ & 0.8713 & 0.9977 \\
\hline & Angle & $100^{0}$ & 0.8713 & 0.9977 \\
\hline & \multicolumn{2}{|c|}{ Length $=3$} & & \\
\hline & Angle & $90^{\circ}$ & 0.6397 & 0.9907 \\
\hline & Angle & $95^{\circ}$ & 0.6048 & 0.9860 \\
\hline & Angle & $100^{\circ}$ & 0.5594 & 0.9860 \\
\hline \multicolumn{3}{|c|}{ Histogram equalisation } & 0.8560 & 0.8721 \\
\hline \multirow{3}{*}{$\begin{array}{l}\text { Gaussian } \\
\text { Noise }\end{array}$} & \multicolumn{2}{|c|}{ Variance $=0.001$} & 0.6446 & 0.9163 \\
\hline & \multicolumn{2}{|c|}{ Variance $=0.005$} & 0.5876 & 0.7233 \\
\hline & \multicolumn{2}{|c|}{ Variance $=0.01$} & 0.5607 & 0.6860 \\
\hline \multirow{4}{*}{$\begin{array}{c}\text { Gamma } \\
\text { intensity } \\
\text { Transforma- } \\
\text { tion }\end{array}$} & \multicolumn{2}{|c|}{ Gamma $=0.7$} & 0.8934 & 0.9488 \\
\hline & \multicolumn{2}{|c|}{ Gamma $=0.8$} & 0.9461 & 0.9721 \\
\hline & \multicolumn{2}{|c|}{ Gamma $=1.2$} & 0.9602 & 0.9860 \\
\hline & Gar & $=1.3$ & 0.9246 & 0.9651 \\
\hline & $\begin{array}{l}\text { let transf } \\
\text { el } 1 \mathrm{DW}\end{array}$ & & 0.8235 & 0.8233 \\
\hline
\end{tabular}

\section{CONCLUSiOnS}

A reversible invisible digital watermarking scheme is proposed in this paper to provide a copyright protection to digital images. The watermark embedding and watermark extraction are done in the discrete wavelet transform domain. In the proposed scheme watermark was embedded in HH subband at 3rd/4th level of DWT. To check the invisibility, histogram of both host and watermarked images are compared on the basis of distribution of various pixels with their different intensities and also by human perception of visualization. It is observed that watermark is invisible after embedding in host image. The reversibility feature is validated by comparing the extracted watermark with original watermark image on the basis of the NC values. And to check the robustness of the proposed scheme, various attacks are performed on the watermarked image. The extracted watermark is compared with original watermark on the basis of the NC values. It is observed that the NC values are closer to 1 in every attacks. Experimental results show that proposed scheme has ability to resist against various attacks. The performance evaluation of the proposed scheme are done by comparing the watermarking at 3rd and 4th level DWT, which gives the result in favour of 4th level DWT. At the 4th level DWT, the proposed scheme is more robust against various attacks. The proposed watermark extraction algorithm is simple and fast. It is also fulfilling the required feature for digital watermarking effectively. However, the scheme proposed in this paper cannot be applied directly on colour images. In future work, more effort will be given on this particular issue. 


\section{REFERENCES}

[1] Jian Liu, Xiangjian He, A review study on digital watermarking, IEEE- International conference on information and communication technologies, pp.337-341, 2005.

[2] Ho Moon, Myung Sohn, Dong Jang, DWT- based image watermarking for copyright protection, Springer-Verlang Berlin Heidelberg-Lecture notes in computer science, pp.490-497, 2005.

[3] Mohammad-Reza Keyvanpour, Farnoosh Merrikh-Bayat, Robust dynamic block based image watermarking in DWT domain, Elsevier GmbH- Procedia computer science, vol.17, pp. 238-242, 2011.

[4] LI Hui-fang, CHANG Ning, CHEN Xiao-ming, A study on image digital watermarking based on wavelet transform, Elsevier GmbH-The journal of China universities of posts and telecommunications, vol. 17 , pp. 122-126, 2010.

[5] Razieh Keshavarzian, Ali Aghagolzadeh, ROI based and secure image watermarking using DWT and arnald map, Elsevier GmbHInternational journal of electronics and communication, vol.70, pp.278-288, 2016.

[6] Thai-son Nguyen, Chin-Chen Chang, Xiao-Qian Yang, A reversible image authentication scheme based on fragile watermarking in discrete wavelet transform domain, Elsevier GmbH-International journal of electronics and communication, vol.70, pp.1055-1061, 2016.

[7] Sangita Zope-Chaudhari, Parvatham Venkatchalam, Krishna Budhiraju, Secure dissemination and protection of multispectral images using crypto-watermarking, IEEE journal of selected topics in applied earth observation and remote sensing, vol.8, no.11, pp.5388-5394, 2015.

[8] Dong Zong, Chun Chen, The study of digital watermarking for protection of multimedia, IEEE 2nd International conference on computing control and industrial engineering, vol.2, pp.304-307, 2011.

[9] Vinita Gupta, Atul Barve, Robust and secured image watermarking using DWT and encryption with QR codes, International Journal of computer applications, vol.100, no.14, pp.33-37, 2014.

[10] A Umaamaheshvari, K Thanushkodi, An elaborative study of digital watermarking technique for watermark protection, IEEEICCTET, pp.526-532, 2014.

[11] Asna Furqan, Manish Kumar, Study and analysis of robust DWT-SVD domain based digital image watermarking technique using matlab, IEEE-International conference on computational intelligence and communication technology, pp.638-644, 2015.

[12] Nasrin Makbol, Be e Khoo, Taha Rassem, Block based discrete wavelet transform-singular value decomposition image watermarking scheme using human visual system characteristics, IET Image process, vol.10, pp.34-52, 2016.

[13] Pratibha Sharma, Shanti Swami, Digital image watermarking using 3 level discrete wavelet transform, Conference on advances in communication and control system, pp.129-133, 2013.

[14] Bhupendra Ram, Digital image watermarking technique using discrete wavelet transform and discrete cosine transform, International journal of advancement in research and technology, vol.2, pp.19-26, 2013.

[15] Nikita Kashyap, G Sinha, Image watermarking using 3-level discrete wavelet transform, I.J.Modern education and computer science, vol.2, pp 50_56, 2012.

[16] Chih-Chin Lai, Cheng-Chih Tsai, Digital image watermarking using discrete wavelet transform and singular value decomposition, IEEE transaction on instrumentation and measurement, vol.59, no.11, pp. 3060-3063, 2010.

[17] Yang Quanli, Cai Yanhang, A digital image watermarking algorithm based on discrete wavelet transform and discrete cosine transform, IEEE International symposium on information technology in medicine and education, pp.1102-1105, 2012.

[18] O Jane , E Elbasi, H lk, Hybrid non blind watermarking based on DWT and SVD, Elsevier- Journal of applied research and technology, vol.12, pp.750 61, 2014.

[19] Wei Song, Xiang-Chun Liu, Guo-sheng Yang, Ren-wei Ding, Study on reversible visible watermarking scheme for digital images, IEEE-International conference on transportation, mechanical, and electrical engineering, pp.2375-2378, 2011.

[20] Priyanka Kulkarni, Altaf Mulani, Robust invisible digital image watermarking using discrete wavelet transform, International journal of engineering research and technology, vol. 4, pp.139-141, 2015.

[21] Preeti Arya, Dherendra Tomar, Deepika Dubey, A review on different digital watermarking techniques, International journal of signal processing image processing and pattern recognition, vol. 8, no.10, pp. 129-136, 2015.

[22] Tien-You Lee, Shinfeng D Lin, Dual watermarking for image tamper detection and recovery, Elsevier-Pattern recognition, vol..41, no.11, pp.3497 06, 2008.

[23] Chun-Chi Lo, Y̌u-Chen Hu, A novel reversible image authentication, Elsevier-Signal Processing, vol.98, pp.174-185, 2014.

[24] Chi-Shiang Chan, An image authentication method by applying hamming code on reaaranged bits, Elsevier-Pattern recognition letters, vol.32, no.14, pp.1679-1690, 2011.

[25] Radu O Preda, Semi-fragile watermarking for image authentication with sensitive tamper localisation in wavelet domain, ElsevierMeasurements, vol.46, no.1,pp. 367-373, 2013.

[26] Jun-Chou Chuang, Yu-Chen Hu, An adaptive iamge authentication scheme for vector quantisation compressed image, Elsevier-J.Vis. Commun. Image R., vol. 22, no.5,pp 440-449, 2011.

[27] Hazem Al-Otum, Semi fragile watermarking for grayscale image authentication and temper detection based on an adjusted expanded bit multiscale quantization based technique, Elsevier-J.Vis.Commun.Image R., vol.25, no.5, pp.1064-1081, 2014. 


\section{AUTHOR PROFILE}
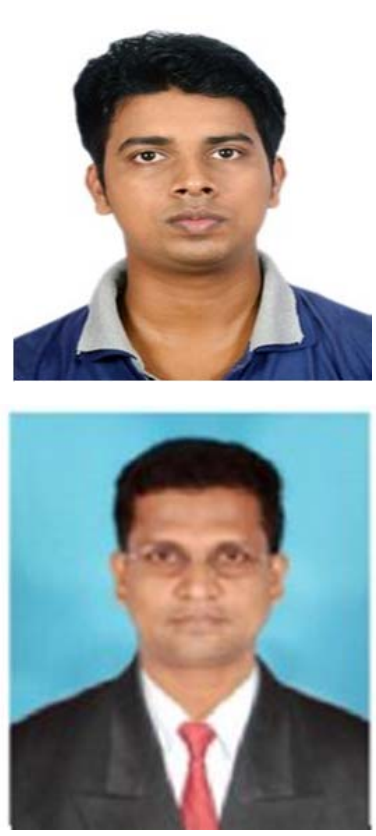

Mayank Mishra was born in Gorakhpur, Uttar Pradesh, India, in 1994. He is final year student of Dual M.Tech in Communication System Engineering at KIIT University, Bhubaneswar, Odisha, India. His current interests are Digital watermarking, Image processing, Digital signal processing and Cryptography.

Email: mmishra1208@gmail.com

Dr. Nirmal Kumar Rout received the B.E. degree in Electronics and Telecommunication Engineering from the University College of Engineering (presently known as Veer Surendra Sai University of Technology), Sambalpur University, Burla, India, in 1991 and the M. Tech degree in computer science from the Utkal University, Bhubaneshwar, India, in 2001. He is awarded with $\mathrm{PhD}$ degree in Electronics and Telecommunication Engineering from the Kalinga Institute of Industrial Technology (KIIT) University, Bhubaneshwar, India, in 2014. From 1993 to 2002, he was a Lecturer with the Department of Electronics and Communication Engineering, Orissa Engineering College, Bhubaneswar. He also served as a Faculty Member of the Institute of Chartered Financial Analysts of India (ICFAI) Institute of Science Technology (ICFAITech), Hyderabad, India, from 2002 to 2007. He is currently working as Professor with the School of Electronics Engineering, KIIT University, Bhubaneshwar, India. He has published number of research papers in various refereed international journals and conferences. His current research interests include active noise control, adaptive signal processing, soft computing, evolutionary computing and image processing. E-mail: routnirmal@rediffmail.com,nkrout@kiit.ac.in. 